Cinémas

Revue d'études cinématographiques

Journal of Film Studies

\title{
Réception critique et contexte : à propos du Déclin de l'empire américain
}

\section{Denise Pérusse}

Volume 2, numéro 2-3, printemps 1992

Cinéma et Réception

URI : https://id.erudit.org/iderudit/1001079ar

DOI : https://doi.org/10.7202/1001079ar

Aller au sommaire du numéro

Éditeur(s)

Cinémas

ISSN

1181-6945 (imprimé)

1705-6500 (numérique)

Découvrir la revue

Citer cet article

Pérusse, D. (1992). Réception critique et contexte : à propos du Déclin de l'empire américain. Cinémas, 2(2-3), 88-106. https://doi.org/10.7202/1001079ar
Résumé de l'article

Cet article se propose d'examiner la façon dont un film est reçu dans un contexte social, culturel et esthétique différent de son contexte d'origine et de mettre en évidence les mécanismes de lecture et d'interprétation qui président lors de sa réception. Il s'agit d'une sorte d'archéologie de la réception qui table sur les éléments contextuels et cognitifs comme facteurs-clés dans la réception-appropriation-interprétation de l'oeuvre. L'appropriation d'un film implique une sorte d'opération de déconstruction/reconstruction du sens dans laquelle sont engagés une vision du monde et un système de références préalablement acquis. Pour esquisser un modèle de lecture, l'auteure prend $L e$ Déclin de l'empire américain de Denys Arcand comme cas de figure et la critique cinématographique française comme médiateur privilégié entre l'oeuvre québécoise et le public. 


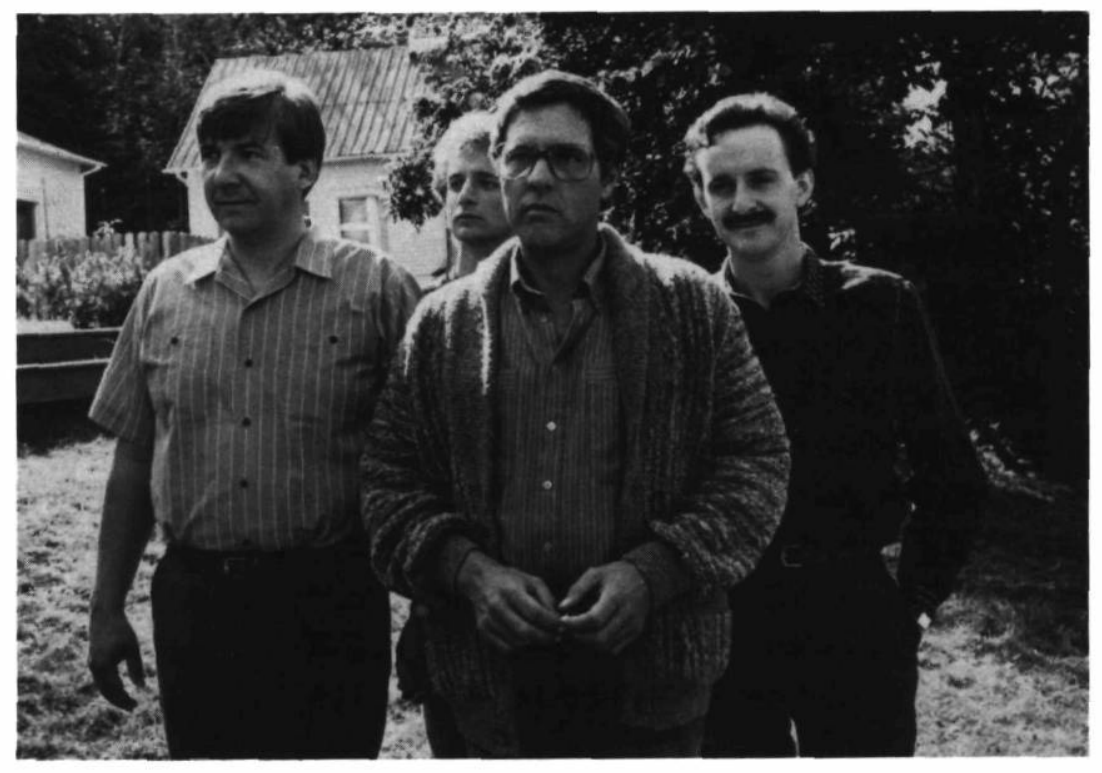

Le Déclin de l'empire américain de Denys Arcand (1986) Coll.: Cinémathèque québécoise 


\title{
Réception critique et contexte: à propos du Déclin de l'empire américain
}

\section{Denise Pérusse}

\section{RÉSUMÉ}

Cet article se propose d'examiner la façon dont un film est reçu dans un contexte social, culturel et esthétique différent de son contexte d'origine et de mettre en évidence les mécanismes de lecture et d'interprétation qui président lors de sa réception. Il s'agit d'une sorte d'archéologie de la réception qui table sur les éléments contextuels et cognitifs comme facteurs-clés dans la réceptionappropriation-interprétation de l'œuvre. L'appropriation d'un film implique une sorte d'opération de déconstruction/reconstruction du sens dans laquelle sont engagés une vision du monde et un système de références préalablement acquis. Pour esquisser un modèle de lecture, l'auteure prend Le Déclin de l'empire américain de Denys Arcand comme cas de figure et la critique cinématographique française comme médiateur privilégié entre l'œuvre québécoise et le public.

\begin{abstract}
This article examines the way a film is received in social, cultural and aesthetic contexts that differ from its context of origin, and the mechanisms of reading and interpretation which govern its reception. It presents a sort of archeology of reception that views contextual and cognitive elements as key factors in the reception/ appropriation / interpretation of a film. The appropriation of a film involves an operation of deconstruction/reconstruction of meaning in terms of a previously acquired worldview
\end{abstract}


and system of references. The authoroutlines the features of this model of reading though a study of the reception of Denys Arcand's Decline of the American Empire in France and the role of film critics there as privileged mediators between this Quebecois film and the French public.

Le pôle de la réception a été une dimension grandement négligée dans le passé au sein des différentes approches théoriques et historiques en cinéma. Par exemple, les approches formalistes ont souvent cantonné le film à ses thèmes et ses formes esthétiques en évacuant sa dimension sociale ou historique et en disqualifiant le rôle du spectateur dans la production du sens ${ }^{1}$. Les approches marxistes ont saisi le film comme une modalité des infrastructures économiques et ont considéré la réception de l'œuvre comme une activité qui se produit selon des normes et des critères préformulés et préétablis. La sociologie traditionnelle, quant à elle, s'est limitée le plus souvent à des enquêtes empiriques sur les publics en tablant sur des caractéristiques sociologiques traditionnelles (sexe, âge, scolarité, etc.), sur une évaluation des goûts cinématographiques, ou encore sur les réactions des publics aux œuvres sans pour autant expliciter l'interaction entre la production, l'œuvre et sa réception.

Ce n'est que récemment que les études en cinéma (sémiotique, historique, sociologique, esthétique de la réception, pragmatique, féministe) ont mis de l'avant un paradigme relativement nouveau qui place le spectateur au centre de la réflexion théorique. Ces études ont fait une brèche notammant dans la clôture sémiotique et ont relocalisé leurs discours sur l'acte de production dans son rapport dynamique avec le spectateur potentiel. On parle désormais du rôle actif du lecteur, du travail du spectateur, de l'activité d'interprétation, de l'expérience esthétique, du spectateur comme foyer réceptif et cognitif. On tente de construire des modèles de la situation de réception qui tiennent compte de facteurs cognitifs, contextuels, historiques.

Dans l'étude suivante, la question du spectatoriat sera abordée en adoptant une approche à la croisée de l'esthétique de la réception ${ }^{2}$, de la sociologie de la connaissance et de la culture, 
approche qui considère le film dans son rapport pragmatique ${ }^{3}$ au spectateur et qui observe à quelle(s) lecture(s) un film a donné lieu selon des époques et des publics différents. Plus précisément, il s'agit d'examiner la façon dont un film est reçu dans un contexte social, culturel et esthétique différent de son contexte d'origine et de mettre en évidence les mécanismes de lecture et d'interprétation qui président lors de sa réception.

Pour esquisser un modèle de lecture, nous avons privilégié $L e$ Déclin de l'empire américain de Denys Arcand comme cas de figure et la critique cinématographique française comme médiateur privilégié entre une œuvre québécoise et le public. Consacré par Le Déclin de l'empire américain qui a connu en France un succès phénoménal en recueillant plus d'un million d'entrées ${ }^{4}$, Arcand est devenu une des figures québécoises les plus saluées par le public français. Au-delà de cette constatation d'ordre quantitatif qui justifie déjà un intérêt pour ses œuvres, il apparaît important d'orchestrer une lecture, une «révision» des jugements de la critique française, de dévider cet écheveau complexe de discours hétérogènes, de jugements normatifs et esthétiques sur lequel se profile une compétence réceptrice. Enfin, le cas du Déclin nous sert de tremplin pour brosser éventuellement un portrait global de la réception critique en Frances. Létude en question ne saurait être considérée comme achevée. Elle s'intègre à l'intérieur d'une recherche plus vaste permettant de mieux saisir l'interaction entre la critique française et les films québécois.

\section{La critique française comme instance médiatrice 6}

Avant d'aborder un modèle de situation de lecture, il importe de préciser les raisons qui ont motivé ce choix d'analyser les jugements portés par la critique cinématographique française sur une œuvre québécoise. Dans Les Films québécois en France ${ }^{7}$, nous avons mis en évidence la performance des œuvres québécoises au guichet français en dégageant les réussites et les hécatombes commerciales, et du même coup les choix du grand public, comme ultime arbitre de la popularité d'une œuvre. Ici, une étude des jugements critiques, de par leur caractère explicite, permet de constater directement ce que ces «superspectateurs» apprécient ou réprouvent des films québécois. Dans cette perspective, les 
critiques peuvent être vus comme des distillats des goûts, des préférences du public, comme des témoignages indicateurs des représentations, images, mythes et préjugés qui travaillent l'imaginaire collectif d'une société à propos d'une autre société.

Notre corpus de base rassemble des textes provenant de quotidiens, revues hebdomadaires ou mensuelles spécialisées ou non en cinéma publiés en France. Les critiques ne sont évidemment pas un public socialement et culturellement homogène. Bien sûr, il pourrait être intéressant de tenir compte, à l'analyse, de la provenance de chaque texte critique, en dégageant l'orientation politique ou esthétique du journal ou de la revue qui détermine dans une certaine mesure le choix des œuvres et la place historique des films. Malgré ces différences évidentes au sein du monde de la critique, notre parti pris est de considérer au départ l'ensemble des textes comme une seule et même trame discursive ${ }^{8}$. En fait, il ne s'agit pas tant d'esquisser une hiérarchie au sein des diverses critiques cinématographiques et de dégager leurs a priori esthétiques et idéologiques, tributaires en partie de l'orientation même des revues ou journaux, que de déconstruire de façon globale le discours de la critique française tenu sur le cinéma québécois. Autrement dit, il nous importe de voir comment la critique sélectionne les informations, construit des réseaux de significations, met en jeu des représentations, réactive au passage la corde intertextuelle en faisant ressurgir la trace de certains textes filmiques, littéraires ou autres, hiérarchise et produit certains paramètres de valorisation esthétiques, classe les films, etc.

\section{Un modèle de lecture}

Il s'agit ici d'une sorte d'archéologie de la réception centrée sur la culture pour produire un modèle de situation de lecture qui table sur les éléments contextuels et cognitifs comme facteurs clés dans la réception-appropriation-interprétation de l'œuvre. Nous partons de l'hypothèse que la réception d'un film implique une sorte d'opération de déconstruction et de reconstruction du sens dans laquelle sont engagées les idéologies d'une société (visions du monde, stéréotypes socioculturels, ensemble de préconstruits, de clichés, etc.) et un système esthétique préalablement acquis (références cinématographiques ou culturelles, normes esthétiques 
en vigueur, etc.). Si les films québécois et le public destinataire d'une autre formation sociale donnée (entendre ici la critique française) sont en possession de répertoires culturels, sociaux et esthétiques distincts, dérivés des idéologies et d'un système esthétique issus de leurs sociétés, il nous apparaît essentiel de mettre en évidence ce moment où il $\mathrm{y}$ a intersection entre le répertoire du spectateur et celui de l'œuvre.

Le modèle de situation de lecture proposé est en grande partie emprunté à celui élaboré par Kathleen McCormick et Gary F. Waller ${ }^{9}$, modèle lui-même grandement influencé par les travaux du phénoménologue Wolfang Iser ${ }^{10}$. Si leur modèle s'applique au départ à l'objet littéraire, il nous semble éclairant de le transposer à l'objet cinématographique. Donnons au départ les principaux postulats sur lesquels s'échafaude leur modèle de situation de lecture.

- L'activité de lecture n'est jamais seulement une activité individuelle ou une expérience purement subjective. Elle doit être décrite d'abord comme une activité cognitive, activité qui apparaît dans un contexte social.

- Tout modèle de lecture doit insister sur la contribution du texte et du lecteur dans l'activité de lecture et sur le fait que le texte comme le lecteur est idéologiquement situé. Bien que le texte soit le lieu sur lequel la production du sens prend place, néanmoins quand un texte est écrit, il est le produit d'idéologies générales et littéraires (ici cinématographiques) dérivées d'une formation sociale donnée.

- La notion d'idéologie est centrale et renvoie à cet ensemble de valeurs, de croyances, de règles, de pratiques qu'une société donnée considère comme «allant de soi», "naturelles» et qui traverse l'ensemble de ses pratiques. Une des fonctions de l'idéologie dans une société est de définir et de limiter les pratiques culturelles de ses individus. Comme l'expliquent McCormick et Waller:

Ideology helps tie us together by giving us seemingly coherent representations and explanations of our social practices, and in particular by giving us the langage by which we describe and thus try to perpetuate them. Thus ideology acts as a kind of social glue, binding us all together (p. 196). 
Cette position rejoint celle avancée par Michel Foucault dans L'Ordre du discours ${ }^{11}$ ou encore celle avancée par Marc Angenot dans ses travaux sur le discours social ${ }^{12}$.

- Si l'idéologie traverse tout texte, le mode d'appropriation de l'idéologie dans un texte donné est appellé répertoire, soit la combinaison spécifique d'idées, de normes, de conventions, de règles qui a permis que le texte soit écrit. Le répertoire du texte peut être scindé en deux: un volet plus spécifiquement littéraire (ici cinématographique) qui renvoie aux éléments formels du texte (du film) - convention, intrigue, forme, mode de narration, construction des personnages, etc. - et un autre plus général ou extra-textuel qui renvoie aux idées véhiculées par le texte (valeurs, croyances, etc.).

Concrètement, dans toute situation de lecture, le répertoire du texte entre en relation avec celui du lecteur. Ce dernier, partie prenante d'une formation sociale donnée, s'approprie également les idéologies d'une société et bricole son propre répertoire. Comme celui du texte, son répertoire est scindé en deux volets. Un volet plus littéraire ou cinématographique concerne ses expériences filmiques antérieures, ses présupposés sur ce qu'est ou doit être le cinéma, ses outils cognitifs ${ }^{13}$, ses stratégies de lecture ${ }^{14}$, etc. Un second volet de son répertoire, plus général, renvoie à l'ensemble de ses croyances, de ses idées sur différents aspects de la réalité (la politique, la religion, l'éducation, le mode de vie, etc. ${ }^{15}$.

C'est donc dans cette interaction entre les répertoires généraux et cinématographiques du spectateur et ceux du texte filmique que l'activité de lecture se met en branle ${ }^{16}$. Voyons maintenant comment ce modèle peut éclairer la réception d'une œuvre québécoise par la critique française.

\section{Le Déclin de l'empire américain: un cas type}

Plusieurs textes critiques sont parus en France sur les œuvres de Denys Arcand: d'abord au cours des années 70 avec la sortie au grand écran de La Maudite Galette, Réjeanne Padovani, Gina; ensuite dans les années 80 avec celle du Crime d'Ovide Plouffe, du Déclin de l'empire américain et finalement de Jésus de Montréal. Compte tenu de l'espace rédactionnel limité, nous avons dû rapidement nous résoudre à jouer du scalpel dans un 
matériel documentaire abondant et nous en tenir ici à la réception du Déclin ${ }^{17}$.

Lorsqu'on lit l'ensemble des textes critiques ${ }^{18}$, au-delà du fait que la plupart font un résumé des principaux enjeux diégétiques — façon la plus commune et la plus facile d'interagir avec le film - et donnent ensuite une appréciation du film, un certain nombre de récurrences ressort nettement:

— la trace d'un certain exotisme géographique;

- l'identification ethnique;

- la mise en contexte du cinéaste en tablant sur ses réalisations antérieures et sa place dans le cinéma québécois;

- l'appel à des stéréotypes socioculturels;

— le recours à des rapprochements cinématographiques ou littéraires;

— un accent sur la forme du récit.

\section{L'exotisme}

Plusieurs critiques ne peuvent s'empêcher de dissoudre l'histoire du Déclin dans un paysage exotique même si le film laisse peu de place à ce genre de clichés géographiques. Tout de même, non seulement situe-t-on précisément l'action du film sur les rives du lac Memphrémagog ${ }^{19}$ — allant jusqu'à parler du «flamboiement des Cantons de l'Est en automne» —, mais encore on vante les «splendeurs» ou «l'atmosphère paradisiaque de l'été indien», les «somptueux paysages qui inciteraient (...) à la sérénité», «les rives du lac Memphrémagog enchâssées d'érables rouges», les «beaux paysages de lac», etc. Parfois, le ton devient carrément lyrique:

(...) il y a tout lieu de penser que le plus bel endroit où l'on puisse attendre la fin du monde soit les rives du lac Memphrémagog dont les eaux miroitantes réflètent l'incendie rougeoyant des essences québécoises, l'automne venu ${ }^{20}$.

Le recours à des appréciations chargées d'exotisme, où l'on table sur le dépaysement, le milieu physique, les espaces naturels, montre bien la fascination qu'ont toujours exercée le vaste continent américain et les espaces sauvages du Québec sur les Français en général. Moins naïvement, il pourrait s'agir d'un moyen utilisé 
par la critique pour rendre ce film québécois centré sur les dialogues plus attrayant au lecteur français. Cela dit, nous sommes loin du syndrome «Maria Chapdeleine» que constatait Jacqueline Gerolds dans son étude sur la réception des romans québécois en France par les critiques littéraires ${ }^{21}$. À l'époque, ceuxci avaient été obnubilés par les grands espaces, les cabanes dans la neige, les chemises à carreaux, etc. Dans le cas du Déclin, la critique française reconnaît que le film va au-delà de dimensions strictement exotiques ou folkloriques.

\section{Lidentification ethnique}

La majorité des critiques utilisent le biais des origines du cinéaste, identification somme toute légitime et justifiable compte tenu de la nécessité de présenter le cinéaste à ses lecteurs potentiels. Règle générale, pour désigner le cinéaste, on sera enclin à utiliser l'épithète canadien alors que pour désigner l'univers diégétique du film, on a recours à l'appellation québécoise: on parle alors d' «intellectuels québécois», de «milieu universitaire québécois», de «frustrés version québécoise», de «profs de facs au Québec», etc. On pourrait s'étonner de l'emploi du terme «canadien» par la critique de l'époque (soit les années 1986-87) qui n'est pas sans savoir que le cinéaste en question est originaire d'une province bien connue pour ses velléités d'indépendance. Par ailleurs, ces épithètes en apparence relativement neutres se chargent dans certains cas de connotations qui ont pour effet de contredire leur relative neutralité.

(...) quand on sait que l'équipe est originaire du Canada francophone, on se dit que, si on assiste peut-être au déclin de l'Empire américain, on vit en revanche l'avènement de l'ère québécoise ${ }^{22}$.

Le terme québécois, s'il est utilisé pour désigner l'univers diégétique, est également le biais pour parler de la langue du film, un facteur qui, selon l'enquête menée par l'Institut québécois du cinéma auprès des distributeurs ${ }^{23}$, constitue dans une certaine mesure une barrière linguistique de taille en France. Donnons la parole à deux critiques: 
Les comédiens d'Arcand sont tous remarquables et ne forcent pas leur accent québécois vers trop de pittoresque. On n'entend qu'un grossier «ciboire!» à la place d'un honnête «merde!». Pour le reste, les mots sont les mêmes. Simplement les Québécois d'Arcand ont la langue mieux pendue et dégourdie que les Français et ne reculent devant rien pour faire comprendre ce qu'ils ont à dire ${ }^{24}$.

L'essentiel est dans le dialogue échangé par des comédiens qui possèdent l'art du naturel et s'expriment en français avec unaccent québécois qui nous fait changer de planète sans pour autant quitter la maison ${ }^{25}$.

Il semble que des moyens ont été pris pour éviter que la langue constitue un fossé infranchissable entre le film et le public français, avec succès d'ailleurs. Le film n'était-il pas sous-titré dans plusieurs cinémas à Paris! On a aussi cherché à rester sur les rails du français international, tout en laissant filtrer au passage un zeste de québécois, question d'alimenter le pittoresque. D'ailleurs, c'est sur la langue plutôt que sur les grands espaces et les paysages - comme nous l'avons vu antérieurement — que la critique joue davantage la carte de l'exotisme.

\section{La mise en contexte du cinéaste}

Si le cinéaste est désigné surtout pour son appartenance canadienne, il est aussi précédé d'une très bonne réputation. La plupart des critiques, à la vue du Déclin, évoquent ses réalisations antérieures, surtout celles des années 70 . On fait une filiation directe avec Réjeanne Padovani qui rappelle, sous un autre registre, le ton cynique et sombre de l'auteur. On mentionne Le Crime d'Ovide Plouffe non pas tant pour la qualité du film que pour l'interprétation d'un de ses comédiens: Gabriel Arcand.

Les films antérieurs d'Arcand ont pavé la voie au Déclin qui répond en quelque sorte aux attentes d'un public familier à son ton «détaché, impérial, très pince-sans-rire, reconnaissable immédiatement». Les points de repères de la critique sont essentiellement liés à l'œuvre d'Arcand. Lorsqu'elle s'aventure sur le terrain de la cinématographie québécoise, on a droit aux succès des années 70. «La faillite des intellectuels québécois, c'est aussi celle du cinéma québécois, dont la vigueur donna il y a quinze ans des espoirs un peu vite oubliés» ${ }^{26}$ ou encore «Le cinéma québécois qui 
fit des ravages dans les années soixante-dix, a pratiquement disparu pour des raisons économiques» ${ }^{27}$, etc.

Vraisemblablement, le répertoire cinématographique de la critique est constitué avant tout d'œuvres québécoises des années 70. On se doute bien que ce sont surtout les films de Carle (Les Mâles, La Mort d'un bûcheron, La Vraie Nature de Bernadette, La Tête de Normande St-Onge) qui ont marqué l'imaginaire français et le hantent encore ${ }^{28}$.

\section{La question de l'intertexte}

Selon Michael Riffaterre, l'intertexte renvoie à «l'ensemble des textes que l'on peut rapprocher de celui qu'on a sous les yeux, l'ensemble des textes que l'on retrouve dans sa mémoire à la lecture d'un passage donné» (p. 5-6). Plus globalement, l'intertexte désigne toutes les structures discursives ou artistiques, antérieures ou contemporaines auxquelles fait allusion le texte et toutes celles qui informent la perception du lecteur.

Si le film regorge de citations qui réfèrent à d'autres textes, à commencer par le titre qui renvoie directement à un ouvrage d'Edward Gibbon ${ }^{29}$ - ce qui d'ailleurs ne manquera pas d'être souligné explicitement ou allusivement par certains critiques , on fait constamment des rapprochements cinématographiques et littéraires. Des phrases du type «On se souvient de», «impossible de ne pas faire référence à», «Le Déclin fait penser à», etc. sont légion. $\mathrm{Si}$, comme stratégie cognitive, la comparaison à des cinéastes ou écrivains bien connus facilite l'accès de l'œuvre au lecteur, elle permet avant tout de clarifier sa propre perception. Dans le cas du Déclin, on se réfère constamment à Sade, en le désignant explicitement ${ }^{30}$ ou allusivement en jouant sur le titre d'un de ses ouvrages bien connus: La Philosophie dans le boudoir $^{31}$. D'autres figures littéraires sont appelées à la barre (Sartre, De Beauvoir, Pierre Kast, etc.) ainsi que des bédéistes bien connus en France (Reïser et surtout Bretecher ${ }^{32}$ ). Dans tous les cas, on est pratiquement toujours branché sur des auteurs français. Du côté cinématographique, l'éventail est plus large. Une filiation presque automatique se fait au départ avec une des œuvres de Woody Allen: Tout ce que vous avez toujours voulu savoir sur le sexe sans jamais oser le demander, comme si le titre de l'un cadrait da- 
vantage avec le sujet de l'autre. Si la vieille filière américaine est également mise à l'ordre du jour avec Joseph Mankiewicz (Chaînes conjugales) ou Joseph Losey (Streamers), la référence aux contes moraux de Rohmer demeure la référence française par excellence. Mais, dans tous les cas, ces rapprochements avec d'autres cinéastes sont là pour évoquer des similitudes ou des points de repères sur le plan cinématographique.

Une bonne part de l'évaluation critique porte sur la forme adoptée par le récit. On s'attarde au style et on s'attend à ce que le cinéaste se plie aux exigences d'un genre. Pour le Déclin, la comédie semble rallier la majorité des critiques. On parle tour à tour de «comédie légère», de «comédie de mœurs très au-dessus du niveau moyen habituel», de la «distribution de personnages [qui] respecte les types de comédie», d' "une des comédies les plus brillantes qu'on ait vues depuis l'âge d'or de Joseph L. Mankiewicz», etc. Par ailleurs, la force du Déclin, c'est aussi de travailler le genre et d'innover en combinant un nouveau genre: le porno «bavard». L'affiche annonçait le film comme une «histoire de cul à voir avec les oreilles»; ne préparait-elle pas le terrain à ce type de réception?

Ce diagnostic de la critique sur la forme du film s'accompagne généralement d'un certain nombre de jugements normatifs ou prescriptifs sur ce qu'est ou doit être le cinéma: «Habituellement au cinéma, on se contente d'exhiber le sexe. Ici, on l'évoque par mille aphorismes qui conjuguent dérision et provocation...» ${ }^{33}$, ou encore:
Àl'évidence, le cinéma c'est d'abord des images. Quand la parole, les dialogues deviennent envahissants, bien souvent le film ne fonctionne pas. Sur un thème qui aurait pu être spectaculairement cinématographique, le sexe, le çnéaste canadien Denys Arcand a pris le parti des mots. Un pari risqué et brillamment réussi ${ }^{34}$.

Encore là, la force d'Arcand réside dans le fait qu'il a su contourner certaines conventions, travailler le genre et faire preuve d'innovations formelles.

\section{La question du référent}

Tout film laisse filtrer des éléments liés à un contexte extrafilmique ou référentiel. La question du référent touche 
nécessairement le spectateur, car l'expérience que suscite chez lui l'acte de réception, loin de se réaliser dans le vide, doit être mise en rapport avec ses propres expériences dans sa vie pratique, avec ses propres valeurs et croyances, etc.

Si la force du Déclin, selon la plupart des critiques, est de passer au crible le monde contemporain occidental, on ne manque pas de comparer des éléments référentiels du film et ceux qui sont propres à la société française. Par exemple, le thème du film sur les relations hommes-femmes est considéré comme très actuel à l'heure des petites annonces du Minitel ${ }^{35}$.

La critique ne s'embarrasse pas toujours de problèmes historiques ou sociologiques posés par le film, soit la place des intellectuels au Québec. Le Québec est ici aplati, poncé, coupé de ses racines, de son contexte d'origine. De plus, une filiation directe est tissée entre Amérique et États-Unis.

Les textes laissent également filtrer un certain nombre des lieux communs ou des généralisations du style: «On sait que les intellectuels sont bavards, les intellectuels québécois semblent battre des records.» ${ }^{36}$ La critique donne également prise à des idées toutes faites sur un Québec libéré de la longue tutelle religieuse. Le spectre de Valérie n'est pas loin!: «Vont-ils [les Européens] se ruer comme l'ont fait les Québécois, alléchés en bonne partie par le titre et les histoires de sexe qu'on y raconte?.» ${ }^{37}$ Par ailleurs, on ne manque pas de considérer cette libéralisation des mœurs affichée avec tant de virulence suspecte: «Quand l'intellectuelle du groupe déclare avoir couché avec le mari d'une de ses copines, lequel trompe celle-ci avec la moitié du Canada. Quelle santé, mes aïeux! Nos cousins québécois ne se vantent-ils pas un peu?.» ${ }^{38}$

Ces quelques exemples n'épuisent évidemment pas le type de réception affiché par la critique. Ils veulent surtout montrer que la critique tend à récupérer dans le film les éléments qui comblent son horizon d'attente et à les subordonner à son système de référence. Elle fouille dans son répertoire (cinématographique ou plus général) pour y trouver un moyen de saisir un film issu d'une autre formation sociale. Dans le cas du Déclin, nous avons vu que la critique table peu sur l'exotisme géographique compte tenu que le film échappe à tout folklore et laisse peu de place à ce type 
d'emprise. Si les points de comparaison sont surtout liés à une tradition littéraire et cinématograhique française, si on mise sur l'universalité du film tout en disséminant au passage des faits de culture propres à la société française ou des idées toutes faites sur le Québec, la bonne réception du film demeure également liée au fait qu'il a su retravailler le genre et le renouveler.

\section{En guise de conclusion}

Il n'y a pas lieu de conclure cette analyse de la réception qui se veut avant tout un work in progress. Nous aimerions simplement revenir sur les visées heuristiques d'une telle démarche. Il nous semble qu'une étude des jugements de la critique comme médiateur par excellence entre l'œuvre et le public est une des voies d'accès au phénomène collectif de la communication cinématographique. De plus, une analyse de la réception telle que nous en avons suggéré les grandes lignes a pour conséquence de réfuter tout structuralisme immanent comme tout sociologisme réducteur. Notre démarche suggère également que l'acte de réception doit être envisagé avant tout comme un processsus interactif, dynamique où le répertoire du spectacteur interagit avec celui du film. Elle suppose aussi que le répertoire du spectateur est extrêmement mouvant au fil de ses rencontres avec les œuvres filmiques.

Enfin cette analyse, ici centrée sur un cas de figure, demeure nécessairement incomplète. Si nous avons tracé quelques balises d'une problématique en pleine expansion, il s'agit maintenant de prendre en considération un corpus beaucoup plus large - en commençant par la réception de l'ensemble des films d'Arcand en France - et de dégager les diverses possiblités d'interface entre le répertoire de la critique française et celui des films québécois. Il faudra voir notamment si la critique choisit d'exploiter les différences, les écarts entre son répertoire et celui du film au point d'oublier le film lui-même ou encore si elle travaille à combler les écarts tout en relocalisant le discours sur le film. Beaucoup de pain sur la planche mais perspective fascinante pour quiconque s'intéresse aux questions de récêption, d'altérité, d'intertextualité et de culture. 


\section{NOTES}

1 Il est certain que les lectures faites par les spectateurs d'un film donné sont en bonne partie influencées par les stratégies inhérentes au film. Mais en pratique, toutes les stratégies textuelles peuvent ne pas être observées ni être actualisées dans une situation de lecture particulière.

2 Dans la foulée des travaux de Hans Robert Jauss, Pour une esthétique de la réception (Paris: Gallimard, 1978). Voir également le modèle proposé par Jügen E. Müller dans le présent volume: «Pragmatique historique du film: Nouvelle Vague et conception de l'auteur. La réception d'À bout de souffle aux Pays-Bas». Ses remarques sur le lecteur, comme disposant d'un «stock spécifique et historique de savoir»-stock qui sert de supportà la réception d'un film, sont éclairantes pour mes propres recherches sur le cinéma québécois et la réception critique.

3 Une pragmatique du cinéma est une méthode interprétative qui vise à rendre compte des rapports entre un texte filmique et les usages qu'on en fait dans un contexte donné (social, culturel, économique, etc.).

4 Dans Les Films québécois en France, nous avons démontré que la structure de l'exploitation en France est pyramidale et repose sur un nombre infime de films qui franchissent la barre du million. Dans le cas des films québécois exploités en territoire hexagonal, Le Déclin del'empire américain avec ses 1,2 millions de spectateurs se classe au $6^{\mathrm{e}}$ rang derrière des coproductions francoquébécoises à gros budgets telles que Quest for Fire, Le Ruffian, Hold-up, Paroles et musique et Au nom de tous les miens. Comme film intégralement québécois, il arrive au premier rang et demeure une figure d'exception compte tenu que le marché cible d'une œuvre québécoise en France (film entièrement produit au Québec ou coproduction dont la participation du Québec est majoritaire) oscille entre 150000 et 200000 spectateurs.

Voir Denise Pérusse avec la participation de Bernard Bérubé, «L'exploitation des films québécois en France selon différentes fenêtres de visibilité» in Les Films québécois en France. Dix ans de cinéma (sous la direction de François Baby et al.) (Montréal, 1991) p. 73-142.

5 Cette réflexion s'inscrit dans la foulée des travaux entrepris par le Centre de recherche sur la réception du cinéma québécois à l'étranger, de l'Université de Montréal.

6 Telle que définie par Jean Cazeneuve dans sa préface à l'ouvrage d'AnneMarie Laulan, Cinéma, presse et public (Paris: Retz-C.E.P. L., 1978) p. 13-17. 
Pour Cazeneuve, une sociologie des massmédia doit «étudier, non plus seulement les émetteurs des messages, les canaux ou les publics, mais aussi et surtout peut-être les médiateurs. C'est là un concept difficile à définir. En un sens, les guides d'opinion sont des médiateurs puisqu'ils façonnent pour autrui la portée des messages diffusés; sont également médiateurs, d'une manière ou d'une autre, tous ceux qui interviennent dans la circulation des messages (...), tous ceux qui renseignent le public sur les émissions ou sur les films» (p. 14$15)$.

7 Les Films québécois en France. Dix ans de cinéma, ibid, p. 73-142.

8 Une première lecture de l'ensemble des critiques publiées sur Arcand montre que la plupart sont relativement brèves (autour d'une page en général) et seules les revues spécialisées en cinéma laissent place à des textes plus élaborés (avec un système de références esthétiques, une rhétorique, et un appareillage critique plus développés).

9 Kathleen McCormick et Gary F. Waller, «Text, Reader, Ideology», Poetics, vol. 16, $\mathrm{n}^{\circ} 2$ (avril 1987) p. 193-208 et Kathleen McCormick, «Task Representation in Writing About Litterature», Poetics, vol. 16, $\mathrm{n}^{\circ} 2$ (avril 1987) p. 131-154.

10 Wolfgang Iser, L'Acte de lecture. Théorie de l'effet esthétique (Bruxelles: Pierre Mardaga éditeur, 1985).

11 Michel Foucault analyse notamment les divers mécanismes et opérations par lesquels l'idéologie d'une société contrôle et délimite les discours. Cf. Michel Foucault, L'Ordre du discours (Paris: Gallimard, 1971).

12 Cf. Marc Angenot, «Intertextualité, interdiscursivité et discours social», Texte 2 (1983) p. 101-112 ou encore son monumental ouvrage: 1889. Unétat du discours social (Longueuil: Éditions du Préambule, 1989).

Pour Angenot, le discours social renvoie à un ensemble des présupposés irréductibles du vraisemblable social ou encore à un ensemble des règles discursives et topiques dans une société donnée sans jamais s'énoncer d'ellesmêmes. De plus, le «discours social au reste ne se manifeste pas nécessairement à la surface rhétorique des textes ou ensemble de textes, mais plutôt dans les soubassements présuppositionnels qui en établissent l'acceptabilité» (1983, p. 106).

13 Par exemple, le mode de sélection et d'organisation de l'information, le fait que certains spectateurs aiment trouver dans un film plusieurs significations alors que d'autres le recoivent plus littéralement, etc.

14 Comme: s'identifier avec les personnages, créer des liens avec d'autres 
films ou d'autres produits culturels, lier sa lecture personnelle du film à des aspects plus larges de sa culture, placer le film dans le contexte de films similaires à ce qu'il a vu, etc.

15 Autrement dit, chaque individu reçoit un film en fonction de sa propre vision du monde et des principes qui règlent les actes de sa vie quotidienne.

16 Un film privilégie ou oriente toujours une certaine lecture tout en jouant sur plusieurs voies pour susciter la curiosité du spectateur. Mais la lecture est avant tout un processus interactif. Tous les spectateurs actualisent les significations du film par un travail de lecture dont les éléments sont issus de leurs propres répertoires. Chacun actualise un film différemment et c'est non seulement attribuable à des facteurs psychologiques ou des traits de personnalité mais surtout à des facteurs culturels - tels des questions de gender, de race, de génération, de culture - qui modifient l'acte de lecture.

17 Les textes ont été recensés dans le cadre des activités du Centre de recherche cinéma/réception, de l’Université de Montréal.

18 Nous avons recensé plus d'une cinquantaine d'articles publiés sur $L e$ Déclin.

19 Donnée géographique reprise du dossier de presse du film.

20 Le Matin (5 février 1987).

21 Cf. Jacqueline Gerolds, Le Roman québécois en France (Paris: Hurtubise $\mathrm{HMH}, 1984)$.

\section{L'Express (6-12 février 1987).}

Ou encore ce titre évocateur du Quotidien de Paris: «Le déclin de l'empire américain. Oh, Canada!» (4 février 1987).

Autre exemple: sil'un des rares reproches adressés au Déclin concerne le choix de son titre, la critique se commet et laisse filtrer son horizon d'attente, sa perception du Québec: «Rien n'est pire au cinéma qu'un titre trompeur.(...) Un spectateur non averti imagine aussitôt un film à message destiné à fustiger l'impérialisme yankee» (Paris Match, 5 février 1987).

23 Malgré que les propos des distributeurs soient souvent contradictoires au sujet de la langue. Voir à ce sujet: Andrée Letendre, Les Films québécois en France, ibid, chapitre 2.

24 Le Monde (5 février 1987). 
26 Le Nouvel Observateur, $\mathrm{n}^{\circ} 1160$ (30 janvier-5 février 1987).

27 Le Parisien (4 février 1987).

28 Gilles Carle demeure une des figures québécoises les plus consacrées en France dans les années 70 tant par la critique que par le grand public. Par exemple, Les Mâles depuis sa sortie en 1971 aurait enregistré près de 300000 entrées, suivi de près par La Vraie Nature de Bernadette avec 281000 entrées depuis sa sortie en 1972 (Voir Denis Bachand, «La réception des films québécois en France», Quebec Studies 9 (1989-1990) p. 69-78).

D'ailleurs ces films reviennent sporadiquement à l'affiche au cours des années 80. C'est dire la réputation qu'a acquis Carle en sol français.

29 Ouvrage édité de nombreuses fois. Voir Edward Gibbon, The Decline and Fall of the Roman Empire (New York: Collier, 1966).

30 Exemples: «Thèse qui n'est pas sans rappeller celle d'Eric von Stroheim et de son maître, le vénérable Marquis de Sade», «ébauche de sujet sadien», «ton de libertinage par trop éloigné de Sade», etc.

31 On parle alors de «confidences hors du boudoir», de «conversations de boudoir", etc.

32 Le Déclin est perçu comme une nouvelle version des «"frustrés" au sirop d'érable».

33 L'Humanité (12 mai 1987).

34 Cinéma 87, n 386 (4-11 février 1987) p. 7.

Ou encore: «Il existe deux façons de réaliser un film à tout petit budget: filmer un décor naturel et les allées et venues d'un pôle à l'autre ou filmer des gens qui discutent dans une pièce. Denys Arcand a choisi le film de conversation. Thème: le sexe. Au cinéma, on le montre, surtout. On en parle rarement» (L'Humanité, 12 mai 1987).

35 «ll y a une parenté entre ce film et les lignes de téléphones roses dont on voit la publicité dans tous les quotidiens" (Cahiers du cinéma , n 393 , mars 1987, p. 48)

36 VSD (5 février 1987).

37 Le Courrier français (novembre 1986). 
38 L'Avant-scène du cinéma, nº 352 (été 1986) p. 89.

\section{OUVRAGES CITÉS}

McCormick Kathleen et Waller, Gary. F. «Text, Reader and Ideology». Poetics, vol. 16, $\mathrm{n}^{\circ} 2$ (avril 1987).

Riffaterre, Michael. «L'Intertexte inconnu». Littérature 41 (1981). 